\title{
Colonel Blotto und seine ökonomischen Anwendungen
}

\author{
Stefan Homburg* \\ Leibniz Universität Hannover
}

\section{Einleitung}

Im heutigen Wohlfahrtsstaat definieren sich die politischen Parteien oft über ihre Positionen zu steuer- und sozialpolitischen Fragen: Welche Steuerlasten sollten die starken Schultern tragen, und welche Transfers sind für die Ärmeren angemessen? Derartige Umverteilungsprobleme dominieren manchen Wahlkampf und stehen im Mittelpunkt dieses Artikels, während Entscheidungen über skalare Politikfragen wie die optimale Größe der Infrastruktur oder Landesverteidigung ausgeklammert werden. Auch geht es im Weiteren nicht um eine normative Fundierung der Steuer-Transfer-Politik, sondern um die positive Analyse der Frage, wie Demokratien umverteilungspolitische Auseinandersetzungen verarbeiten.

Brennan und Buchanan (1980, S. 21) formulierten hierzu in ihrem bekannten Werk „The Power to Tax“ ein „Prinzip der Ausbeutung der maximalen Minderheit“, wonach Demokratien in der Steuer- und Sozialpolitik dazu neigen, eine Minderheit zugunsten der Mehrheit auszubeuten. Die dahinter stehende Idee sei anhand eines einfachen Zahlenbeispiels mit zwei politischen Parteien illustriert, die um drei Wähler werben. Haben die Parteien 300 Geldeinheiten in Form von Steuersenkungen oder Transfererhöhungen zu verteilen, könnten sie jedem Wähler 100 Geldeinheiten anbieten, also mit einem Tripel $(100 ; 100 ; 100)$ in den Wahlkampf ziehen. Bietet aber eine Partei das Tripel (150; 150;0) an, wird sie zwei Stimmen gewinnen, weil die ersten beiden Wähler von diesem Angebot stärker profitieren als von der Gleichverteilung (100; 100; 100), während die an der Gleichverteilung festhaltende Partei nur die Stimme des dritten Wählers erhält und damit die Wahl verliert. Analog gehen bei fünf Wählern zwei leer aus, bei sieben Wählern drei usw.

Das Prinzip der Ausbeutung der maximalen Minderheit hat in der Literatur wenig Widerhall gefunden, weil es auf einem Denkfehler beruht: Zwar schlägt das Politikangebot (150; 150; 0) die Gleichverteilung, doch verliert es zum Beispiel gegen das Angebot $(0 ; 151 ; 149)$, das seinerseits etlichen Alternativen unterliegt, wie man leicht nachprüft. Somit fragt es sich, welche Strategie eine Stimmen maximierende Partei verfolgen sollte, wenn sie ein festes Budget auf mehrere Wähler verteilen kann, jeder Wähler nur an sich denkt und die Partei damit rechnen muss, dass auch ihre Konkurrentin nicht von gestern ist.

Zur Analyse des vorstehend formulierten Problems eignet sich das Colonel Blotto Spiel. Dieses Spiel ist keine jüngere Entdeckung. Es findet sich tatsächlich bereits im ältesten spieltheoretischen Aufsatz überhaupt, nämlich in Borel (1921), und

\footnotetext{
*Korrespondenzadresse: Institut für Öffentliche Finanzen, Leibniz Universität Hannover, Königsworther Platz 1, 30167 Hannover, e-mail: homburg@fiwi.uni-hannover.de. Der Autor dankt seinen Institutsmitarbeitern, einem anonymen Gutachter und dem Herausgeber Lars Feld herzlich für konstruktive Verbesserungsvorschläge.
} 


\section{Stefan Homburg}

dürfte neben dem Gefangenendilemma zu den für ökonomische Anwendungen nützlichsten Spielen gehören. Im Kontrast zu dieser Behauptung steht der geringe Bekanntheitsgrad des Spiels, der wohl darauf beruht, dass seine Lösung ungleich schwieriger ist als die des Gefangenendilemmas und deshalb gut 80 Jahre auf sich warten ließ. Mit diesem Artikel soll das Colonel Blotto Spiel ein wenig bekannter gemacht und sollen einige seiner faszinierenden ökonomischen Implikationen verdeutlicht werden.

\section{Das Colonel Blotto Spiel}

Seinen Namen erhielt das Spiel durch Gross und Wagner (1950), die in einem Forschungspapier des „Rand Project“ der U.S. Air Force folgende militärische Konfliktsituation betrachteten und nach einem fiktiven Colonel Blotto benannten: Der Colonel und sein Gegner verfügen über A bzw. B Bataillone, die sie auf n Schlachtfelder schicken können. Jedem ist die Stärke des Gegners bekannt. Im Morgengrauen entscheiden sich der Colonel und sein Gegner für eine bestimmte Verteilung der Bataillone auf die Schlachtfelder, und es gewinnt derjenige das Schlachtfeld $\mathrm{i}=1 \ldots \mathrm{n}$, der dort mehr Bataillone hingeschickt hat; bei Gleichstand gewinnt keiner. Das Ziel beider Kombattanten besteht darin, möglichst viele Schlachtfelder zu gewinnen.

Formal seien $\mathrm{A}, \mathrm{B}$ und $\mathrm{n}$ ganze Zahlen und $\mathrm{A} \leq \mathrm{B}$. Im Colonel Blotto Spiel $\mathrm{CB}$ $(A, B, n)$ wählt der erste Spieler einen Vektor $\left(x_{1}, \ldots, x_{n}\right)$ mit $x_{1}+\cdots+x_{n}=A$, und der zweite wählt $\left(\mathrm{y}_{1}, \ldots, \mathrm{y}_{\mathrm{n}}\right)$ mit $\mathrm{y}_{1}+\cdots+\mathrm{y}_{\mathrm{n}}=\mathrm{B}$. Dabei sind $\mathrm{x}_{\mathrm{i}}$ und $\mathrm{y}_{\mathrm{i}}$ nichtnegative Zahlen und entweder reell oder ganz. Die Auszahlung des ersten Spielers beträgt

$$
\mathrm{H}=\#\left\{\mathrm{i} \mid \mathrm{x}_{\mathrm{i}}>\mathrm{y}_{\mathrm{i}}\right\}-\#\left\{\mathrm{i} \mid \mathrm{x}_{\mathrm{i}}<\mathrm{y}_{\mathrm{i}}\right\},
$$

wobei das Nummernzeichen die Anzahl der Elemente der Menge bezeichnet. Die Auszahlung des zweiten Spielers beträgt -H; es handelt sich also um ein ZweiPersonen-Nullsummenspiel.

Das eingangs geschilderte Szenario des Werbens zweier Parteien um drei Wähler entspricht dem Colonel Blotto Spiel CB (300; 300; 3), bei dem beide Streitparteien gleich stark sind. Allgemein erlaubt das Spiel unterschiedliche Spielstärken, wobei der zweite Gegner ohne Beschränkung der Allgemeinheit als der Stärkere angesehen wird. In den vergangenen Jahren wurde das Colonel Blotto Spiel weitgehend gelöst, und einige Lösungen sollen im übernächsten Abschnitt präsentiert werden. Zuvor aber wird die Schwierigkeit des Spiels an einem weiteren Beispiel veranschaulicht und hernach, zur Hinführung auf die Lösung, ein verwandtes Spiel betrachtet.

Zur Illustration betrachte man das Spiel CB $(6 ; 6 ; 3)$, bei dem jeder Spieler sechs Bataillone (oder Geldeinheiten) auf drei Schlachtfelder (oder Wähler) zu verteilen hat. Dafür gibt es 28 verschiedene Möglichkeiten, nämlich die Verteilungen (6; 0; $0),(5 ; 1 ; 0),(4 ; 2 ; 0),(4 ; 1 ; 1),(3 ; 3 ; 0),(3 ; 2 ; 1),(2 ; 2 ; 2)$ und ihre Permutationen. Jede Verteilung ist eine reine Strategie im spieltheoretischen Sinn. Man sieht nun leicht, dass es unter diesen reinen Strategien bessere und schlechtere gibt: Eine Konzentrationsstrategie wie $(6 ; 0 ; 0)$ erzielt gegen sämtliche andere Strategien bestenfalls einen Gleichstand, etwa gegen $(4 ; 2 ; 0)$. Ansonsten unterliegt sie, etwa gegen $(0 ; 2 ; 4)$ oder gegen $(2 ; 2 ; 2)$. Damit sind Konzentrationsstrategien die schlechtesten Züge. 


\section{Colonel Blotto und seine ökonomischen Anwendungen}

Etliche weitere Strategien werden von bestimmten anderen Strategien in dem Sinne dominiert, dass sie ihnen und ihren Permutationen teils unterliegen, teils Gleichstand erzielen, aber nie gewinnen. So wird $(5 ; 1 ; 0)$ von $(3 ; 3 ; 0),(3 ; 2 ; 1)$ und $(2 ; 2 ; 2)$ dominiert, $(4 ; 1 ; 1)$ von $(2 ; 2 ; 2)$, $(3 ; 2 ; 1)$ von $(3 ; 3 ; 0)$ und $(2 ; 2 ; 2)$ von $(3 ; 3 ; 0)$. Schließlich verliert die Strategie $(3 ; 3 ; 0)$ gegen $(4 ; 1 ; 1)$ in zwei von drei Fällen.

Es verbleiben allein die Strategie $(4 ; 2 ; 0)$ und ihre Permutationen. Sie werden von keiner anderen Strategie dominiert, sondern gewinnen gegen jede andere Strategie mindestens so oft, wie sie verlieren. Gleichwohl taugen auch diese Strategien nicht als Siegertyp, denn $(4 ; 2 ; 0)$ unterliegt $(0 ; 3 ; 3)$, und auch jede ihrer Permutationen findet ihren Meister. Diese Intransitivität des Colonel Blotto Spiels führt zu einem negativen und gleichwohl beeindruckenden ersten Resultat: Abgesehen von Trivialfällen besitzt das Spiel kein Gleichgewicht in reinen Strategien. Weil es sich aber um ein simples Zwei-Personen-Nullsummenspiel handelt, existieren laut von Neumann (1928) Gleichgewichte in gemischten Strategien. Hierbei kündigen die Spieler lediglich Wahrscheinlichkeitsverteilungen an, statt klar zu sagen, was sie tun werden.

Bezogen auf Umverteilungspolitiken, insbesondere die Steuer- und Sozialpolitik, ergibt sich folgende Schlussfolgerung: Die Sehnsucht der Wähler und Leitartikler nach Politikern, die sagen, was sie meinen und tun, was sie sagen, muss in der Demokratie unerfüllt bleiben. Zwar ist die Existenz solcher Politiker nicht schlechterdings ausgeschlossen, doch werden sie im demokratischen Wettbewerb unterliegen und nach einiger Zeit in Vergessenheit geraten. Einer Partei abzuverlangen, dass sie vor der Wahl klar sagt, was sie im Amt tun wird, ähnelt der naiven Forderung an einen Schere-Stein-Papier Spieler, seine nächsten Züge jeweils anzukündigen. Wer als Politiker so unklug ist, sich auf irgendeine der obigen reinen Strategien festzulegen, etwa auf die Gleichverteilung $(2 ; 2 ; 2)$, wird die Wahl gegen einen versierten Gegner mit Sicherheit verlieren.

Die Einsicht, bei Umverteilungspolitiken seien nur gemischte Strategien optimal, führt zu einem tieferen Politikverständnis und gibt Anlass zur Umwertung mancher Werte. So erscheint der Bundestagswahlkampf 2009 der CDU, die dabei nicht ansatzweise zu erkennen gab, was sie im Fall ihres Wahlsiegs zu tun gedenke, aus dieser Sicht fast genial. Und man versteht die Zurückhaltung der Politikprofis gegenüber konkreten zahlenmäßigen Festlegungen etwa auf einen bestimmten Steuertarif oder bestimmte Transferhöhen - Festlegungen, wie sie die Wissenschaft so liebt. Die Nichtoptimalität reiner Strategien verlangt von den politischen Akteuren, erratisch zu handeln und bisweilen das Gegenteil dessen zu tun, was die Öffentlichkeit zuvor erwartete. Man denkt sofort an den Falken Nixon, der den Vietnamkrieg beendete, die Taube Kennedy, der diesen Krieg begann, an den großen Winston Churchill, den Goebbels „ganz unberechenbar" fand, aber auch an den sozialdemokratischen Kanzler Schröder, der den Spitzensteuersatz beträchtlich senkte oder an die Unionskanzlerin Merkel, die diesen Steuersatz erhöhte.

Bis hier zusammengefasst ist das Colonel Blotto Spiel intransitiv und führen reine Strategien zum Untergang. Damit besteht eine Verwandtschaft zum Schere-SteinPapier Spiel. In beiden Fällen müssen die Spieler randomisieren, um erfolgreich zu sein. Der Schere-Stein-Papier Spieler tut gut daran, jede seiner drei Möglichkeiten rein zufällig mit Wahrscheinlichkeit 1/3 auszuwählen. Die optimalen Strategien des Colonel Blotto Spielers sind kniffliger und erfordern eine Zwischenüberlegung, die im folgenden Abschnitt eingeschoben wird.

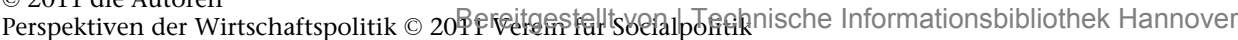




\section{Stefan Homburg}

\section{Das doppeltkontinuierliche Colonel Blotto Spiel}

Ganzzahlige Optimierungsaufgaben sind meist komplexer als ihre reellen Pendants. Diese Tatsache hat Myerson (1993) zu einer bemerkenswerten Vereinfachung des Colonel Blotto Spiels veranlasst. Bildlich gesprochen sind in seinem Modell einerseits die verfügbaren Truppen beliebig teilbar, und andererseits gibt es überabzählbar unendlich viele Schlachtfelder. Es handelt sich also um ein doppelt kontinuierliches Colonel Blotto Spiel. Konkret betrachtet Myerson einen symmetrischen Zwei-Parteien-Wahlkampf, dessen Gegenstand Umverteilungspolitiken bilden. Die identischen Wähler werden durch ein Zahlenkontinuum repräsentiert. Jede Partei kann den Wählern bindende Steuersenkungen bzw. Transfers anbieten, im Mittel aber nicht mehr als b Geldeinheiten; b repräsentiert also das verfügbare Budget pro Wähler. In reinen Strategien besitzt auch diese Variante des Spiels keine Lösung: Bietet die erste Partei zum Beispiel jedem Wähler den Betrag b an, was der Gleichverteilung entspricht, könnte die zweite mühelos die Wahl gewinnen, indem sie zwei Dritteln der Wählerschaft das Eineinhalbfache von b offeriert. Die letztere Strategie kann ebenfalls ohne große Mühe geschlagen werden.

Bei gemischten Strategien bieten die Parteien $\mathrm{zu}$ Beginn des Wahlkampfs gleichzeitig und unabhängig voneinander kumulierte Wahrscheinlichkeitsverteilungen F bzw. G auf der Menge der nicht-negativen reellen Zahlen an. Mit $\mathrm{x}$ als individuellem Wahlkampfangebot muss $\int \mathrm{xf}(\mathrm{x}) \mathrm{dx}=$ $\int \mathrm{xg}(\mathrm{x}) \mathrm{dx}=\mathrm{b}$ gelten, wobei $\mathrm{f}$ und $\mathrm{g}$ die zugehörigen Dichtefunktionen symbolisieren, weil die Angebote im Mittel dem Wert b entsprechen. Vor der Wahl zieht jeder Wähler in den örtlichen Wahlkampfbüros zwei Offerten gemäß den Verteilungen F und G. Anschließend wählt er die erste Partei, wenn sie ihm mehr geboten hat, und sonst die zweite Partei. Man errechnet nun leicht den

$$
\text { Stimmenanteil der zweiten Partei }=\int_{0}^{\infty} \mathrm{F}(\mathrm{x}) \mathrm{g}(\mathrm{x}) \mathrm{dx} \approx \sum \mathrm{F}\left(\mathrm{x}_{\mathrm{i}}\right) \mathrm{g}\left(\mathrm{x}_{\mathrm{i}}\right) \text {. }
$$

In der zur Veranschaulichung aufgeführten Diskretisierungrechts zeigt die Wahrscheinlichkeitsmasse $\mathrm{g}\left(\mathrm{x}_{\mathrm{i}}\right)$ den Anteil der Wähler, dem die zweite Partei den Vorteil $\mathrm{x}_{\mathrm{i}}$ verspricht. Weil $\mathrm{F}\left(\mathrm{x}_{\mathrm{i}}\right)$ jenem Anteil der Wähler entspricht, dem die erste Partei weniger oder gleich viel bietet, stimmen diese $100 \mathrm{~F}\left(\mathrm{x}_{\mathrm{i}}\right) \mathrm{g}\left(\mathrm{x}_{\mathrm{i}}\right)$ Prozent der Wähler für die zweite Partei. Aufsummiert über alle Vorteile erhält man den gesamten Stimmenanteil der zweiten Partei.

Aus diesen Prämissen folgt: Im einzigen Gleichgewicht verteilen beide Parteien ihre Angebote uniform auf dem Intervall [0, 2b]. Auf diesem Intervall nehmen die Verteilungsfunktionen die Werte $F(x)=G(x)=x / 2 b$ an, während sie links davon verschwinden und rechts davon konstant gleich Eins sind. Die Optimalität dieser gemischten Strategie lässt sich leicht beweisen: Spielt nämlich die erste Partei diese Strategie und die zweite Partei eine ganz beliebige Strategie, dann beträgt der Stimmenanteil der zweiten Partei

Stimmenanteil der zweiten Partei $=\int_{0}^{\infty} F(x) g(x) d x \leq \int_{0}^{\infty} \frac{x}{2 b} g(x) d x=\frac{1}{2 b} \int_{0}^{\infty} x g(x) d x=\frac{1}{2}$. 


\section{Colonel Blotto und seine ökonomischen Anwendungen}

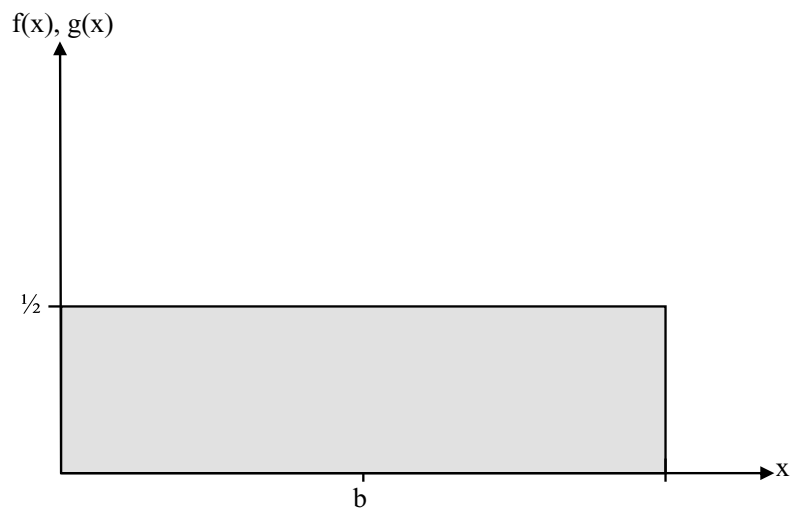

Abbildung 1 Optimale gemischte Strategie.

Die Ungleichung beruht darauf, dass $\mathrm{F}(\mathrm{x})$ auf dem Intervall $[0,2 \mathrm{~b}]$ den Wert $\mathrm{x} / 2 \mathrm{~b}$ annimmt und rechts dieses Intervalls den Wert $1<\mathrm{x} / 2 \mathrm{~b}$. Das Gleichheitszeichen rechts beruht auf der Voraussetzung $\int \mathrm{x} g(\mathrm{x})=\mathrm{b}$. Also beträgt der Stimmenanteil der zweiten Partei nicht mehr als $1 / 2$. Weil die zweite Partei im behaupteten Gleichgewicht die Hälfte der Stimmen erhält, kann sie durch Spielen einer anderen Strategie nichts gewinnen.

Militärisch gewendet lautet das Erfolgsrezept: Verteile deine Truppen ex ante uniform, und schicke auf kein Schlachtfeld mehr als das Doppelte der durchschnittlichen Truppenzahl. Abbildung 1 veranschaulicht die optimalen gemischten Strategien. Intuitiv leuchtet das Ergebnis sofort ein: Man verteilt seine Truppen uniform, um dem Gegner keinerlei Blöße zu geben. Weil negative Truppenzahlen nicht zulässig sind, die Null also eine natürliche Untergrenze bildet, führt dies notwendig auf eine uniforme Verteilung zwischen Null und dem Doppelten des Mittelwertes, symmetrisch um b. Verteilt der erste Spieler seine Truppen in dieser Weise, lohnt es sich für den zweiten nicht, Offerten größer als 2b zu machen: Weil der erste Spieler höchstens $2 b$ bietet, bringen höhere Angebote keine zusätzlichen Siege, kosten aber Ressourcen, die an anderer Stelle fehlen.

Politisch interpretiert lässt sich aus dem Ergebnis die Mahnung herauslesen, nicht viele Versprechungen auf eine kleine Wählerschar zu konzentrieren, sondern jedem höchstens das Doppelte der durchschnittlichen Segnung in Aussicht zu stellen. Die Null bildet für die Politik keine natürliche Untergrenze, weil Steuererhöhungen ebenso zulässig sind wie Steuersenkungen. Dieser Gesichtspunkt ist aber unwichtig. Können die Parteien die Steuern nämlich um einen gewissen Maximalbetrag erhöhen (der sich aus wirtschaftlichen oder rechtlichen Gesichtspunkten ergibt), ersetzt man im obigen Modell einfach die Null durch diesen Maximalbetrag mit negativem Vorzeichen, und das Ergebnis gilt mutatis mutandis fort.

Interessanter - und durchaus schockierend - ist ein anderer Aspekt der politischen Interpretation: Ausgehend von einer homogenen Wählerschaft erzeugt der demokratische Prozess aus sich heraus Ungleichheiten, ist also entgegen landläufiger Ansicht nicht egalitätsfördernd, sondern egalitätsfeindlich. Die Wahrscheinlichkeitsverteilungen F und G muten zwar ex ante egalitär an, doch wird nach der Wahl abgerechnet: Manche Wähler erhalten Vorteile bis zur Höhe 2b, während andere leer ausgehen. Damit wird Gleichheit in Ungleichheit 


\section{Stefan Homburg}

verwandelt. Nun sind die Wähler in der Realität zwar nicht gleich, doch ändert das nichts an der Bedeutung des Resultats. Ist die Wählerschaft nämlich heterogen und gibt es im Ausgangspunkt ein Steuer-Transfer-System, das diese Heterogenität berücksichtigt, dann werden „windfall gains“ nicht, wie es richtig wäre, gleichmäßig auf Steuerzahler und Transferempfänger verteilt, sondern zur Schaffung einer künstlichen Ungleichheit eingesetzt, wobei es vom Zufall abhängt, wer gewinnt bzw. verliert.

Illustrationen liegen auf der Hand. Man denke an die Bundestagswahl 2009, in deren Nachgang Hoteliers zur allgemeinen Überraschung einen Umsatzsteuernachlass von zwölf Prozent erhielten. Seither rechnen Hotels die Übernachtungen zum ermäßigten Satz von sieben Prozent ab, während die in ähnlicher Lage befindlichen Gastronomen für ihre Speisen meist 19 Prozent Umsatzsteuer anzusetzen haben. Die Abgrenzung mutet vollkommen willkürlich an, ist aber beileibe kein Einzelfall. Das gesamte Einkommensteuergesetz besteht aus einer Aneinanderreihung von Ungleichbehandlungen, die sich niemandem so recht erschließen: Landwirte zahlen bei gleichem Gewinn weniger Steuern als andere Unternehmen ( $\S 13 \mathrm{a}$ EStG) und müssen keine Bücher führen; Gutachten zu konkreten Schiffsunglücken lösen Gewerbesteuerpflicht aus, Abhandlungen über prinzipielle Unglücksursachen aber nicht; Arbeitnehmer werden für Tag- bzw. Nachtarbeit unterschiedlich besteuert, Unternehmer aber wiederum unabhängig von der Verrichtungszeit. Ein einfaches Steuersystem „ohne Ausnahmetatbestände“ mag zwar normativ wünschenswert sein, doch wird jede Partei, die dieses Ziel als reine Strategie verfolgt, die Wahl verlieren und ihr Vorhaben nicht umsetzen können.

Auf der Transferseite kann man ähnliches beobachten. So wurde durch die im Vorfeld der Bundestagswahl 2009 geplante Opel-Rettungsaktion eine homogene Betroffenengruppe auseinander gerissen: Arbeiter bei Opel zählten plötzlich mehr als Arbeiter anderer Unternehmen, die nicht gerettet werden sollten. Auch die Dauersubventionen für Landwirtschaft, Bergbau etc. bedeuten eine Privilegierung bestimmter Personen gegenüber anderen, die sich in vergleichbaren Lagen befinden. Alle diese Ungleichheiten lassen sich normativ auf Grundlage einer „veil of ignorance“ Sicht kaum rechtfertigen. Sie sind auch nicht wirklich beliebt. Das obige Modell bietet eine mögliche Erklärung, warum solche Ungleichheiten aus dem politischen Prozess heraus entstehen und Bestand haben: Die Politik randomisiert, was unter Erfolgsgesichtspunkten durchaus ratsam ist. Glücklich, wer dann am Wahltag das große Los zieht, wie zuletzt die Hoteliers - obgleich die Politik hier wohl übers Ziel hinausgeschossen ist und mehr als das Doppelte des Durchschnitts geboten hat, denn in der Nachspielzeit riesiger Bankenrettungsund Konjunkturprogramme wird die durchschnittliche Beglückung in dieser Legislaturperiode eher negativ sein. Gegen die hier behauptete Zufälligkeit der Vergünstigung für Hoteliers könnte man zwar einwenden, dass letztere eifrig für den Wahlkampf gespendet haben. Gespendet haben aber auch andere, wie Energieversorger und Luftverkehrsunternehmen, die nunmehr durch unerwartete Steuererhöhungen das Hotelprivileg mitfinanzieren müssen.

Zusammengefasst vereinfacht Myerson das Ursprungsproblem, indem Ganzzahligkeitsbeschränkungen entfallen und die Budgetbeschränkung nur noch im Erwartungswert erfüllt sein muss. Daher können die Angebote an die Wähler unabhängig voneinander getätigt werden. Optimale Strategien sind in diesem Modell durch Uniformität der Verteilung gekennzeichnet; darüber 


\section{Colonel Blotto und seine ökonomischen Anwendungen}

hinaus wird niemandem mehr als das Doppelte des durchschnittlichen Vorteils versprochen.

\section{Lösungen}

Zurück zur Ausgangsfrage, dem Colonel Blotto Spiel mit endlich vielen Schlachtfeldern. Roberson (2006) hat das Spiel für reellwertige Strategieräume und auf Basis der Annahme, dass bei gleichen Truppenstärken der zweite Spieler obsiegt, weitgehend gelöst. Zur Darstellung seiner Lösungen sei $b=B / n$ die durchschnittliche Truppenstärke des stärkeren Spielers, also die Truppenstärke, die sich bei einer Gleichverteilung auf die Felder ergibt. Gemischte Strategien sind jetzt n-dimensionale Wahrscheinlichkeitsverteilungen auf den Mengen aller nichtnegativen n-Tupel, deren Quersummen A bzw. B ergeben. Im Weiteren werden einige Lösungen des Spiels skizziert, dabei aber nur die Kernideen wiedergegeben und nicht alle Verästelungen und Ganzzahligkeitsprobleme erschöpfend behandelt.

Sind zunächst beide Spieler gleich stark $(A=B)$, beschicken sie jedes Schlachtfeld mit einer auf dem Intervall [0, 2b] uniform verteilten Truppenstärke. Wie im Modell von Myerson erweist es sich als optimal, die Truppenstärken zufällig auszuwählen und auf kein Schlachtfeld mehr als das Doppelte der durchschnittlichen Truppenstärke zu senden.

Es sei nun angenommen, dass der erste Spieler schwächer ist als der zweite $(\mathrm{A}<\mathrm{B})$, jedoch über mindestens $2 \mathrm{~b}$ Truppen verfügt, also $\mathrm{A} \geq 2 / \mathrm{n} \mathrm{B}$ gilt. Bei drei Feldern erreicht der Schwächere mindestens 2/3 der Spielstärke des Gegners, bei vier Feldern ist er mindestens halb so stark usw. Unter dieser Voraussetzung verhält sich der stärkere Spieler so wie im symmetrischen Fall; er verteilt seine Truppen überall uniform auf dem Intervall [0, 2b]. Der schwächere Spieler aber wechselt zu einer Guerillataktik, indem er ein oder mehrere zufällig ausgewählte Felder unbesetzt lässt und seine Truppen auf den übrigen Feldern uniform verteilt. Abbildung 2 zeigt die korrespondierenden Randverteilungen auf einem beliebig ausgewählten Schlachtfeld i. Dabei repräsentiert G die uniforme Strategie des stärkeren Spielers. Der schwächere Spieler setzt gemäß der Verteilungsfunktion F eine positive Wahrscheinlichkeitsmasse auf den Punkt Null und spielt rechts davon uniform, und zwar bemerkenswerterweise auf dem gesamten Intervall [0, 2b]. Frei nach Clausewitz geht der schwächere Spieler dort, wo er überhaupt antritt, mit voller Kraft gegen den Stärkeren vor und gleicht dies aus, indem er einen Teil der Felder ganz aufgibt.

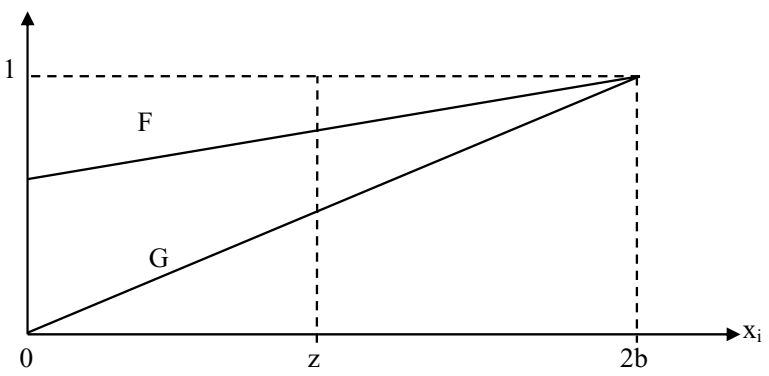

Abbildung 2 Optimale Strategien ungleicher Gegner. 


\section{Stefan Homburg}

Interessant ist hierbei vor allem die Guerillataktik des schwächeren Spielers. Eine intuitive Erklärung dieses Verhaltens gewinnt man durch Interpretation des Colonel Blotto Spiels als einer Menge miteinander verbundener All Pay Auctions; das sind Auktionen, bei denen alle Bieter für ihre Gebote bezahlen, nicht nur der Meistbietende. All Pay Auctions werden etwa zur Analyse des Lobbying herangezogen, einer Aktivität, die auch dann etwas kostet, wenn sie nicht von Erfolg gekrönt wird. Die darauf bezogene Literatur zeigt, dass es für schwache Bieter optimal sein kann, einer Auktion fernzubleiben, wenn sie von der Existenz stärkerer Bieter wissen. Ähnlich verhält es sich hier: Im Wissen um den stärkeren Gegner gibt der schwächere so viele Schlachtfelder von vornherein auf, dass er auf den verbleibenden mit vergleichbarer Stärke zu spielen vermag.

Eine bestrickende politische Deutung ergibt sich aus diesen Überlegungen, wenn man die Schlachtfelder als Politikfelder wie Wirtschaft, Umwelt, Sicherheit oder Familie ansieht. Nach Voraussage der Theorie bestückt eine große oder Volkspartei sämtliche Politikfelder, während eine kleine oder Klientelpartei manche Politikfelder unbeackert lässt und ihre Kräfte auf wenige konzentriert. Gemein ist beiden Parteitypen, dass sie ihre Ankündigungen unscharf halten und sich nicht in die Karten schauen lassen.

Die vorstehend beschriebenen Lösungen betrafen Spiele mit reellwertigen Truppenzahlen. Hart (2008) hat unlängst einige Lösungen für die schwierigste Version des Spiels entwickelt, bei der nur ganzzahlige Bataillone auf die Schlachtfelder verteilt werden; diese Lösungen sind allerdings nicht eindeutig. Das einführend betrachtete Spiel CB (6; 6; 3) besitzt folgende Lösung: Beide Spieler wählen alle Permutationen der Truppenverteilung (4; 2; 0) mit gleicher Wahrscheinlichkeit. Die Analogie $\mathrm{zu}$ den Ergebnissen Myersons und Robersons ist offensichtlich, weil (2; 2; 2) der Gleichverteilung entspricht $(b=2)$, die Spieler nirgends mehr als das Doppelte dieses Durchschnittswerts setzen und die Randverteilungen symmetrisch um den Wert b liegen, wie in Abbildung 3 dargestellt.

Abschließend seien die Lösungen des von Brennan und Buchanan aufgeworfenen Problems präsentiert, 300 Geldeinheiten auf drei Wähler oder Politikfelder zu verteilen. Sind die Geldeinheiten beliebig teilbar, dann wählt jede Partei Tripel (x, y, z) mit der Quersumme 300, deren Elemente jeweils uniform auf dem Intervall [0, 200] verteilt sind. Demnach erhält jeder Wähler durchschnittlich



Abbildung 3 Optimale Bataillonszahl auf dem i-ten Schlachtfeld. 


\section{Colonel Blotto und seine ökonomischen Anwendungen}

100 Geldeinheiten, und die maximale Minderheit der Wähler wird mit Wahrscheinlichkeit Null ausgebeutet. Gleichwohl erzeugt das Politikspiel eine beachtliche Ungleichheit der ursprünglich gleichen Wähler.

Darf die Geldsumme nur ganzzahlig aufgeteilt werden, formt jede Partei eine $101 \times 3$-Matrix, deren Spalten (mit Zeilenindex $i=0 \ldots 100$ ) den Bildungsgesetzen $2 \mathrm{i},(100+2 \mathrm{i})$ modulo 202 und (200-4i) modulo 202 gehorchen; dabei bezeichnet modulo den nach Division verbleibenden Rest. Jede Zeile der so konstruierten und weiter unten abgebildeten Matrix zeigt eine zulässige reine Strategie, weil die Quersumme stets 300 ergibt. Im Gleichgewicht spielt jede Partei alle sechs Permutationen dieser 101 reinen Strategien mit Wahrscheinlichkeit 1/606. Erneut erweist sich gründliches Randomisieren als politisch überlebenswichtig, für jeden Wähler wird symmetrisch um den Durchschnittswert gespielt, und nie wird mehr als das Doppelte des Durchschnittswerts gesetzt.

$$
\left(\begin{array}{ccc}
0 & 100 & 200 \\
2 & 102 & 196 \\
& \ldots & \\
198 & 96 & 6 \\
200 & 98 & 2
\end{array}\right)
$$

\section{Schluss}

Das Medianwählertheorem hat in der ökonomischen Theorie der Politik seit langem einen festen Platz. Dieses Theorem kann auf Meinungsverschiedenheiten bezüglich skalarer Größen angewandt werden - wie den optimalen Umfang der Bundeswehr, die optimale Infrastrukturausstattung und dergleichen. In diesem Fall ist die Annahme eingipfliger Präferenzen plausibel, die Menge der Politikoptionen wird bezüglich der Wählerstimmen transitiv, enthält damit ein maximales Element, und im Ergebnis spielen die konkurrierenden Parteien reine Strategien, die der Meinung des Medianwählers entsprechen.

Viele politische Angelegenheiten besitzen jedoch eine andere Struktur, die man als Umverteilungsproblem im weitesten Sinn des Wortes bezeichnen kann. Hierzu gehört die Steuer-Transfer-Politik, aber auch ganz allgemein die Besetzung verschiedener Politikfelder, die um dieselben Ressourcen konkurrieren. Bei derartigen mehrdimensionalen Entscheidungen sind die Präferenzen regelmäßig nicht mehr eingipflig. So mag jeder Wähler eine bestimmte Steuer-Transfer-Politik vorziehen, die ihn besonders begünstigt. Betrachtet der Wähler Politikangebote, die ihm denselben Vorteil verheißen und sich nur in der Behandlung anderer Wähler unterscheiden, als gleichwertig, dann entfallen Eingipfligkeit und Transitivität, und reine Strategien sind unter den Annahmen des Medianwählertheorems nicht länger optimal.

Um diesem Dilemma zu entrinnen, wurde die Theorie probabilistischen Wählens entwickelt; gute Darstellungen finden sich bei Coughlin (1992) oder Grüner (2008). Dem Ansatz unterliegen die Annahmen, Wählerpräferenzen seien der Politik nicht genau bekannt und Wähler machten bei Abstimmungen Fehler. Unter diesen Voraussetzungen können Gleichgewichte existieren, obwohl die Wählerpräferenzen nicht eingipflig sind. Allerdings reicht die Annahme der Unsicherheit über Wählerpräferenzen und Wahlverhalten für sich genommen 


\section{Stefan Homburg}

nicht aus. Vielmehr hat Kirchgässner (2000) gezeigt, dass Gleichgewichte nur unter bestimmten Konkavitäts- bzw. Konvexitätsvoraussetzungen existieren, die ihrerseits empirisch und theoretisch fragwürdig sind.

Eine radikale Alternative $\mathrm{zu}$ den bestehenden Ansätzen ist das Colonel Blotto Spiel. Dieses Spiel eignet sich zur Analyse mehrdimensionaler Entscheidungen, insbesondere Umverteilungsentscheidungen, und charakterisiert Gleichgewichte in gemischten statt in reinen Strategien. Bisher fristete Colonel Blotto ein rechtes Schattendasein, mutmaßlich deshalb, weil das Spiel erst in den vergangen Jahren gelöst wurde und die Struktur seiner Lösungen ein grundlegendes Umdenken erfordert. Mit diesem Artikel sollte das Spiel ein wenig bekannter gemacht und angeregt werden, es in den Kanon der ökonomischen Theorie der Politik aufzunehmen und zur Analyse wirtschafts- und finanzpolitischer Probleme heranzuziehen. Hierfür sprechen die faszinierenden Ergebnisse, die sich mit relativ geringem Aufwand herleiten lassen und $\mathrm{zu}$ einem besseren Politikverständnis, beitragen:

Hierbei ist zuerst und zuoberst die Einsicht zu nennen, dass reine Strategien bei Umverteilungskonflikten versagen und insofern alle Rufe nach einer konsistenten Politik vergebliche Liebesmühe sind. Diese Botschaft steht in diametralem Gegensatz zum Medianwähleransatz und der Theorie probabilistischen Wählens, die beide auf reine Strategien fokussieren. Politikpraktiker scheinen diesen Gesichtspunkt viel besser zu verstehen als Wissenschaft und Medien. Während der Leitartikler nämlich fragt, warum ein Politiker wie Horst Seehofer - der ebenso gut zur F. D. P. gehören könnte wie zur Linkspartei - im politischen Wettkampf zu überleben vermag, zeigt das Colonel Blotto Spiel, dass gerade Unberechenbarkeit und erratisches Verhalten strategische Stärken sind, die im politischen Alltag zum Erfolg verhelfen.

Zweitens führt das Spiel zu einigen konkreten Empfehlungen: Bei gleich starken Spielern sollte jeder die Wähler oder Politikfelder gleich intensiv pflegen, seine Einsätze jeweils uniform verteilen und nirgends mehr als das Doppelte seiner durchschnittlich verfügbaren Ressourcen einsetzen. Ein schwächerer Spieler tut demgegenüber gut daran, einen Teil der Politikfelder aufzugeben und auf den verbleibenden dieselbe gemischte Strategie zu spielen wie der Stärkere. Andererseits bleiben viele Fragen für weitere Forschung offen: Gelingt es einer Partei besser als der anderen, die Wähler über die durchführbaren Politiken zu täuschen, nehmen die Siegchancen der erstgenannten Partei zu; anders ausgedrückt sind A und B nicht notwendig unabhängig von den gespielten Strategien. Zweitens mag ein allzu erratisches Verhalten im politischen Wettbewerb Stimmen kosten, weil es die Glaubwürdigkeit beschädigt. Während ein Colonel beliebig zwischen den Truppenverteilungen randomisieren kann, ist dies einer Partei nicht unbedingt möglich. Dieser Gesichtspunkt könnte die Ablösung des ZweiParteien-Systems durch das Aufkommen kleiner Parteien erklären: Sie beackern nach Art der Guerillataktik kleinere Politikfelder, und die Unsicherheit wird von der Ankündigung bestimmter Politiken auf die Koalitionsbildung verlagert, wobei die Kunst darin besteht, möglichst viele Koalitionsvarianten offen zu halten.

Eine Literaturauswahl zum Colonel Blotto Spiel umfasst über die bisher genannten Arbeiten hinaus Adamo und Matros (2009), Laslier und Picard (2002), Lizzeri (1999) oder Kovenock und Roberson (2009). Neben spieltheoretischen Lösungen, die bei versierten Gegnern angezeigt sind, wird zunehmend auch 


\title{
Colonel Blotto und seine ökonomischen Anwendungen
}

das tatsächliche Spielverhalten experimentell untersucht; vgl. etwa Arad und Rubinstein (2009) oder Chowdhury et al. (2009).

\section{Literatur}

Adamo, T. und A. Matros (2009), A Blotto Game with Incomplete Information, Economics Letters 105, 100-102.

Arad, A. und A. Rubinstein (2009), Colonel Blotto's Top Secret Files, Working Paper, Tel Aviv University.

Borel, E. (1921), La théorie du jeu et les équations intégrale à noyau symétrique. Übersetzung: The Theory of Play and Integral Equations with Skew Symmetric Kernels, Econometrica 21 (1953), 97-100.

Brennan, G. und J.M. Buchanan (1980), The Power to Tax. Cambridge University Press, Cambridge.

Chowdhury, S.M., D. Kovenock und R.M. Sheremeta (2009), An Experimental Investigation of Colonel Blotto Games, CESifo Working Paper Series No. 2688, München.

Coughlin, P. (1992), Probabilistic Voting Theory. Cambridge University Press, Cambridge.

Gross, O. und R. Wagner (1950), A Continuous Colonel Blotto Game, RAND Research Memorandum 408.

Grüner, H. P. (2008), Wirtschaftspolitik. Springer, Berlin, Heidelberg et al. 2001, 3. Auflage 2008.

Hart, S. (2008), Discrete Colonel Blotto and General Lotto Games, International Journal of Game Theory 36, 441-460.

Kirchgässner, G. (2000), Probabilistic Voting and Equilibrium: An Impossibility Result, Public Choice 103, 35-48.

Kovenock, D. und B. Roberson (2009), Inefficient Redistribution and Inefficient Redistributive Politics, Public Choice 139, 264-272.

Laslier, J. und N. Picard (2002), Distributive Politics and Electoral Competition, Journal of Economic Theory 103, 106-130.

Lizzeri, A. (1999), Budget Deficits and Redistributive Politics, Review of Economic Studies 66, 909-928.

Myerson, R. (1993), Incentives to Cultivate Favored Minorities Under Alternative Electoral Systems, American Political Science Review 87, 856-869.

Neumann, J. von (1928), Zur Theorie der Gesellschaftsspiele, Mathematische Annalen, 295320.

Roberson, B. (2006), The Colonel Blotto Game, Economic Theory 29, 1-24.

\begin{abstract}
Redistributional issues are important in contemporary welfare states. These issues cannot be analyzed using the median voter theorem because preferences fail singlepeakedness: Collective preferences are intransitive, giving rise to cyclical preferences. A suitable instrument for analyzing redistributional issues is the Colonel Blotto game. This game is older than the more familiar prisoner's dilemma, but it has been solved only recently. The article introduces the Colonel Blotto Game as well as the general structure of its solutions. Thereafter, the game's logic is illustrated using several policy examples. The two most fascinating results state that, in a political contest, it is never optimal to use pure strategies, and that the political process itself induces remarkable inequalities.
\end{abstract}

